\title{
A Pyrimidine Auxotroph of Sclerotinia sclerotiorum for Use in Biological Weed Control
}

\author{
By R. VINCENT MILLER, $†$ EUGENE J. FORD, NINA J. ZIDACK $\ddagger$ AND \\ DA VID C. SANDS* \\ Department of Plant Pathology, Montana State University, Bozeman, Montana 59717-0002, \\ $U S A$
}

(Received 10 October 1988; revised 6 March 1989; accepted 20 March 1989)

\begin{abstract}
An auxotroph of Sclerotinia sclerotiorum, A1-pyr, was isolated after mutagenesis of ascospores with ultraviolet light. Addition of cytosine and to a lesser extent uracil, but not thymidine, to minimal medium permitted growth of this auxotroph. Protoplasts of A1-pyr failed to regenerate on Czapek agar medium without cytosine supplementation $\left(20 \mathrm{mg} \mathrm{l}^{-1}\right)$; on cytosinesupplemented medium $0 \cdot 1 \%$ of protoplasts successfully regenerated. The mutant was avirulent on four of seven susceptible hosts unless an exogenous cytosine source was applied at the inoculation site. The requirement for external cytosine makes Al-pyr a potential candidate for use in biological weed control. While isolates of this fungus obtained from nature attack numerous beneficial crop and native plants, Al-pyr would be limited to the area of cytosine application, thus reducing the threat to beneficial plants.
\end{abstract}

\section{INTRODUCTION}

Many host-specific pathogens such as the rusts lack the lethality needed for effective biological control of weeds. Conversely, many highly lethal pathogens attack large numbers of plants, often including crops and beneficial native species. This has created a problem in the search for plant pathogens that are lethal enough for effective weed control but which do not endanger non-target plants. Consistent with this problem, our search to find effective biological agents to control Canada thistle (Cirsium arvense (L.) Scop.) and spotted knapweed (Centauria maculosa Lam.) in Montana disclosed that the pathogen with the most potential for effective weed control was also one of the most non-specific plant pathogens known, Sclerotinia sclerotiorum (Lib.) deBary (Adams, 1974; Purdy, 1979). The potential of this organism for weed control was demonstrated in limited field trials where strains of the fungus reduced populations of both weed species by over $80 \%$ (Broston \& Sands, 1986; unpublished data, our laboratory). Instead of seeking an alternative, more specific pathogen, we chose to try a genetic approach to render $S$. sclerotiorum environmentally safe. The fact that this pathogen is reported to attack weeds in over 40 plant genera (Adams, 1974; Adams et al., 1974, 1983; Bodine, 1935; Klisiewiczk 1986; Watson et al., 1974; Morgan, 1971) is another incentive to render this fungus into an acceptable biological control agent.

The approach was to use mutagenesis and selection to generate strains of the fungus with reduced host ranges, with reduced abilities to survive (e.g. non-sclerotium formers), or in some way restricted to the area of application. Both spontaneous (Garrabrandt et al., 1983; Huang, 1981, 1983) and induced (Blanchette \& LeTourneau, 1977; LeTourneau 1984; Wyllie \& Walter,

\footnotetext{
† Present address: Mycogen Corporation, 3303 McDonald Avenue, Ruston, Louisiana 71270, USA.

$\ddagger$ Present address: Department of Plant Pathology, 139 Funchess Hall, Auburn University, Auburn, Alabama 36849-5409, USA.
} 
1957) mutants of $S$. sclerotiorum have been described. Using $p$-fluorophenylalanine and gamma irradiation LeTourneau obtained mutants of the fungus that were resistant to fluorophenylalanine, others that had altered colony pigmentation, and still others unable to form sclerotia (Blanchette \& LeTourneau, 1977; LeTourneau, 1984). Wyllie \& Walter (1957) described morphological, colour and pathogenicity variants of $S$. sclerotiorum induced by irradiation. The biochemical bases of these mutations were not determined in these previous studies. However, some of the mutants may have been auxotrophs, as nutrition has been shown to affect pathogenicity of a number of both plant and animal pathogens (Bacon et al., 1951, Boone, 1971; Boone et al., 1957; Garber, 1954a, b; Garber et al., 1956; Kline et al., 1957; Tinline, 1963).

The purpose of this research was to genetically improve $S$. sclerotiorum as a weed control agent. Here we report isolation of an auxotrophic mutant that may be limited to the application site by virtue of its nutritional requirement.

\section{METHODS}

Fungal isolates and cultivation. The wild-type isolate (KA2) of S. sclerotiorum used in this study was originally collected in 1982 by C. G. Eayre and B. S. Brosten from a diseased spotted knapweed near Missoula, Montana. The fungus was grown on potato dextrose agar (PDA) and stored for up to a year as air-dried sclerotia or as infested white proso millet (Panicum miliaceum $\mathrm{L}$.) at $4{ }^{\circ} \mathrm{C}$. The auxotroph was grown on PDA supplemented with either $2 \mathrm{~g}$ yeast extract $\mathrm{l}^{-1}$ or $50 \mathrm{mg}$ cytosine $\mathrm{l}^{-1}$ and again stored as sclerotia or infested millet that had imbibed an aqueous solution of yeast extract or cytosine. Minimal agar medium was Czapek solution agar (Difco) amended with asparagine and thiamin (Wong \& Willetts, 1973). To prepare infested millet, the dry grain was autoclaved for $1 \mathrm{~h}$ at $121^{\circ} \mathrm{C}$, then a sterile nutrient solution $\left(1.5 \mathrm{~g} \mathrm{l}^{-1}\right.$ each of yeast extract and Casamino acids; or cytosine $)$ and antibiotics (300 mg streptomycin sulphate $1^{-1}$ and $75 \mathrm{mg}$ tetracycline hydrochloride $\mathrm{l}^{-1}$ ) was added at rates of $750 \mathrm{ml}$ per $\mathrm{kg}$ dry grain. The sterile millet was inoculated with a $7 \mathrm{~mm}$ diameter mycelial agar disc of $S$. sclerotiorum; the fungus was allowed to grow for $14 \mathrm{~d}$ at room temperature, air dried at $27^{\circ} \mathrm{C}$, and stored at $4{ }^{\circ} \mathrm{C}$.

Ascospore production. Fungal cultures were grown on PDA or PDA supplemented with yeast extract (PDY agar) for 2 to 3 weeks at $20^{\circ} \mathrm{C}$, inducing sclerotium formation and maturation. The sclerotia were collected aseptically, washed with sterile distilled water for $48 \mathrm{~h}$ at $4{ }^{\circ} \mathrm{C}$, dried for $72 \mathrm{~h}$ under a laminar-flow hood, and placed on $2 \%$ (w/v) water agar (purified agar, Difco) following the protocol of Henson \& Valleau (1940). Apothecial development of Montana isolates of this fungus required a vernalization period (Saito, 1977) of eight weeks at $4^{\circ} \mathrm{C}$. Those Petri plates with sclerotia developing apothecial stipes were then placed in a $16^{\circ} \mathrm{C}$ growth chamber with a $12 \mathrm{~h}$ per day photoperiod (fluorescent lighting) to maturate apothecial discs (Saito, 1977).

Mutagenesis. Ascospores from mature apothecia were discharged into sterile distilled water and then onto PDA plates (approximately 2500 spores per plate). The plates with ascospores were placed $16 \mathrm{~cm}$ from a $254 \mathrm{~nm}$ ultraviolet light source (General Electric G8T5, 15 W source) and irradiated for $80 \mathrm{~s}$, which permitted about 2\% ascospore survival. Surviving ascospores were germinated and transferred to fresh PDA agar.

Detection and characterization of auxotrophs. Mycelial agar plus $(7 \mathrm{~mm})$ were cut from 3- to 5-d-old irradiated fungal cultures growing on PDA. The plugs were placed on minimal agar medium supplemented with yeast extract. Isolates requiring yeast extract for growth, indicating auxotrophy, were isolated and characterized for nutritional requirements by auxanography (Holliday, 1956).

Fungal protoplasts. Protoplasts of Al-pyr and the wild-type were produced by the Novozyme 234/cellulase wall digestion procedure of Stanway \& Buck (1984). Protoplast regeneration was determined on minimal agar or supplemented minimal agar $\left(1 \mathrm{~g}\right.$ yeast extract $1^{-1}$ or $20 \mathrm{mg}$ cytosine $\left.\mathrm{1}^{-1}\right)$ amended with $1.2 \mathrm{M}$-sorbitol as stabilizer.

Pathogenicity tests. Crowns of host plants (see Fig. 4) were inoculated with wild-type or mutant isolates of the fungus with either $7 \mathrm{~mm}$ diameter mycelial agar plugs or infested millet in a modification of the procedure of Price \& Colhoun (1975). All host plants were one week old at the time of inoculation except sweet clover and lettuce, which were two weeks old. External nutrient sources (yeast extract or cytosine) were applied at the time of inoculation by either mixing an aqueous nutrient solution with the inoculum or by pipetting the nutrient solution onto a cotton plug at the inoculation site.

\section{RESULTS}

Isolation of mutant Al-pyr. Three isolates, from over 1100 mutagenized ascospore isolates screened, failed to grow on minimal agar. Of the three, only A1-pyr retained its auxotrophic character over subsequent generations. The auxotrophic phenotype was separated from nonauxotrophic sectors of fungal colonies by hyphal tipping followed by selection for the nutritional requirement. The mutant phenotype was stabilized without re-occurrence of prototrophic phenotypes after the third hyphal tipping. 


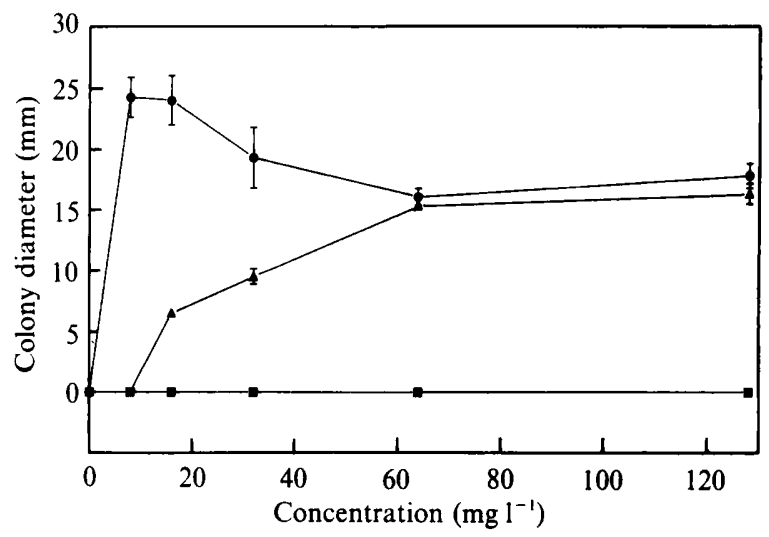

Fig. 1. Radial growth of $S$. sclerotiorum mutant A1-pyr on a modified Czapek medium supplemented with cytosine $(\mathbf{O})$, uracil $(\boldsymbol{A})$, or thymidine $(\boldsymbol{\square})$. Mean and standard deviation of three replications.
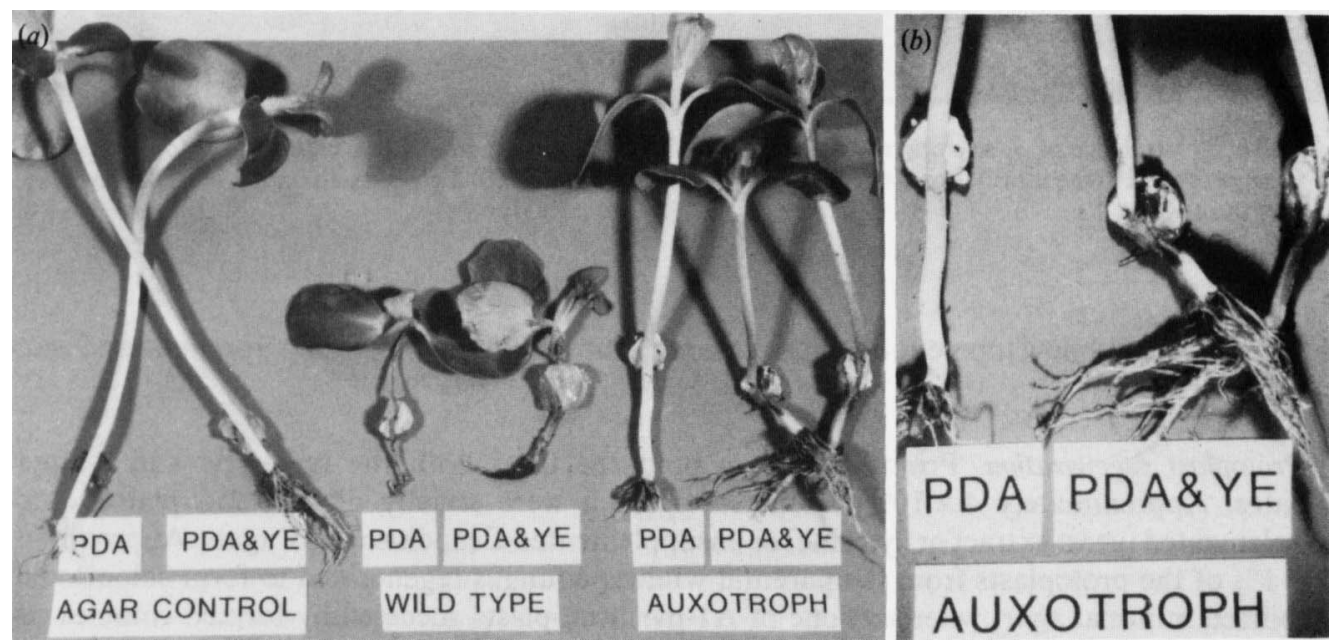

Fig. 2. Restoration of virulence of $S$. sclerotiorum Al-pyr by yeast extract. A comparison of agar controls with wild-type $S$. sclerotiorum and auxotroph Al-pyr grown on PDA or PDA supplemented with yeast extract (YE) is shown in $(a)$, with a close-up of the Al-pyr lesion site in $(b)$. For the YEsupplemented inocula, a solution of $2 \mathrm{mg} \mathrm{YE} \mathrm{ml}^{-1}$ was dripped over the inoculation site.

The nutritional requirement of A1-pyr was determined by auxanography (Holliday, 1956) to be for pyrimidines. Fig. 1 shows the relative growth rates of the mutant on minimal medium as a function of cytosine, thymidine, or uracil supplements. Only cytosine and, to a lesser extent, uracil complemented the nutritional requirement of the mutant. Similarly, Al-pyr infestations of inoculated millet seed, greater than $20 \%$, required nutrient supplementation as indicated by fungal growth from seed plated onto PDA. A supplement of 10,30 and $100 \mu \mathrm{g}$ cytosine $1^{-1}$ to the millet at time of inoculation resulted in 22,80 and $100 \%$ infestation, respectively.

Stability of the mutant phenotype. Reversions of the auxotroph to prototrophy have never been observed during the numerous laboratory transfers of A1-pyr. In addition, of 264 single A1-pyr ascospores tested, all exhibited the auxotrophic phenotype. Stipe formation appeared normal 


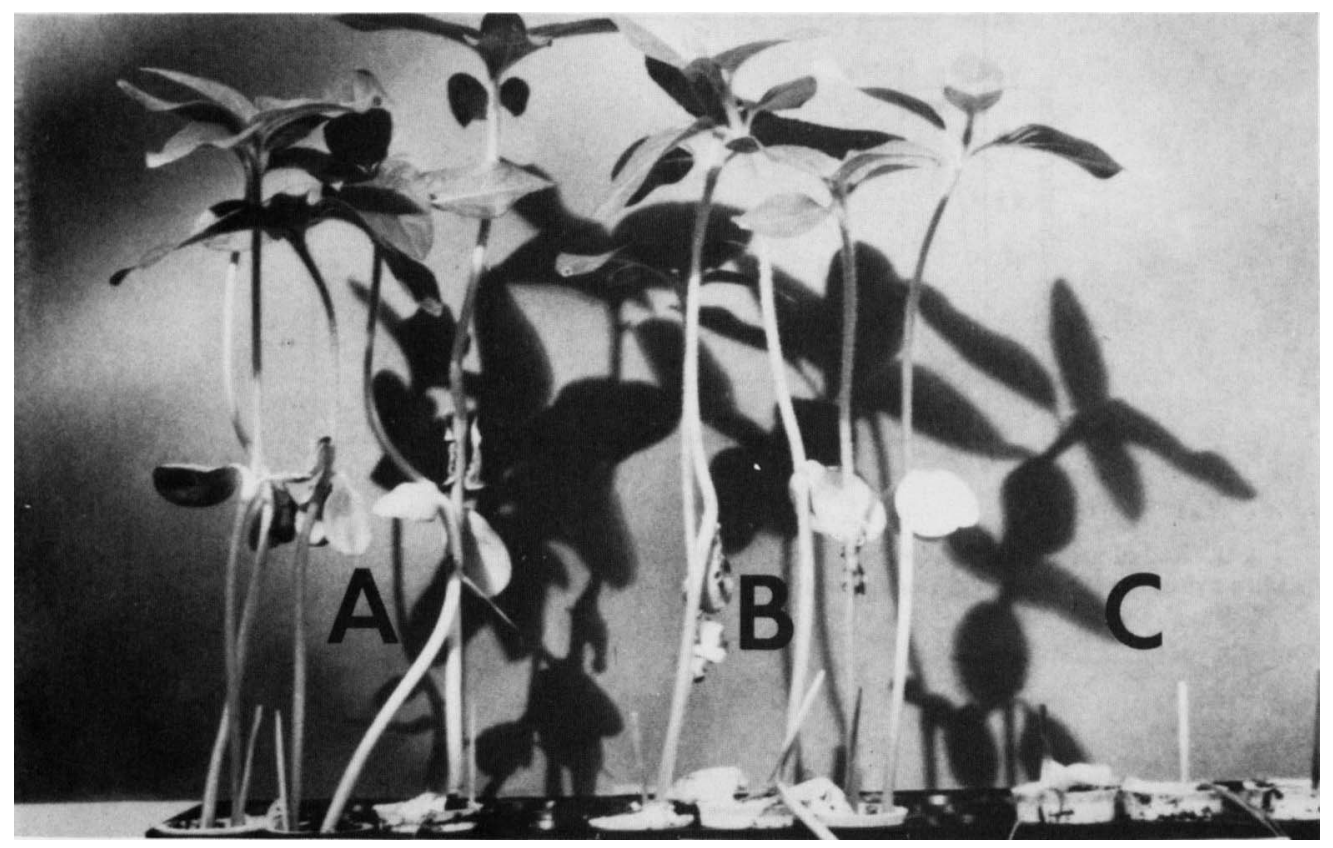

Fig. 3. Virulence of $S$. sclerotiorum A1-pyr on sunflower seedlings from PDA cultures. Cotton plugs were applied to the inoculation site containing (A) distilled water, (B) 2 g yeast extract $\mathrm{l}^{-1}$, or (C) $50 \mathrm{mg}$ cytosine $1^{-1}$.

during apothecial development of the auxotroph, but less than $1 \%$ of the stipes matured into apothecial discs.

Protoplast regeneration. Protoplasts were prepared from both the wild-type and A1-pyr isolates. Approximately $4 \times 10^{6}$ protoplasts of each were serially diluted and plated onto supplemented (yeast extract or cytosine) or unsupplemented Czapek/sorbitol agars. Approximately $1 \%$ of the protoplasts from the parental wild-type fungus regenerated to form colonies on stabilized minimal agar, whereas none of A1-pyr protoplasts successfully formed colonies on unsupplemented agar. A regeneration rate (as colony-forming units) of $0.1 \%$ was observed for Al-pyr on medium containing cytosine, whereas only microscopic germ-tubes formed on medium containing yeast extract. In all of the tests, protoplasting was verified as water controls failed to produce colonies.

Effect on virulence. Virulence of Al-pyr was determined by inoculating plants with either mycelial agar discs (PDY agar) or infested millet. The fungus failed to induce a lesion on any sunflower seedlings when applied as a mycelial disc unless a nutrient supplement was applied together with the inoculum (Fig. 2). Fig. 3 shows regained virulence of A1-pyr on sunflower with external applications of a yeast extract solution $\left(2 \mathrm{mg} \mathrm{ml}^{-1}\right)$ compared with a cytosine solution $\left(50 \mu \mathrm{g} \mathrm{ml}^{-1}\right)$, applied to the inoculation site on cotton plugs. The effect of nutrients, applied to cotton plugs or mixed with millet inoculum, on virulence of A1-pyr was determined on seven hosts (Fig. 4). Lettuce and clover were the most susceptible, succumbing to disease even without nutrient supplements (Fig. 4). The remaining species occasionally developed slight lesions when inoculated without external nutrients but they were never observed to die in these conditions. The necessity of nutrient supplementation for complete infestation of the millet and resulting residual pyrimidines presumably accounts for the enhanced virulence with the millet-based inoculum (Fig. $4 b$ ). 

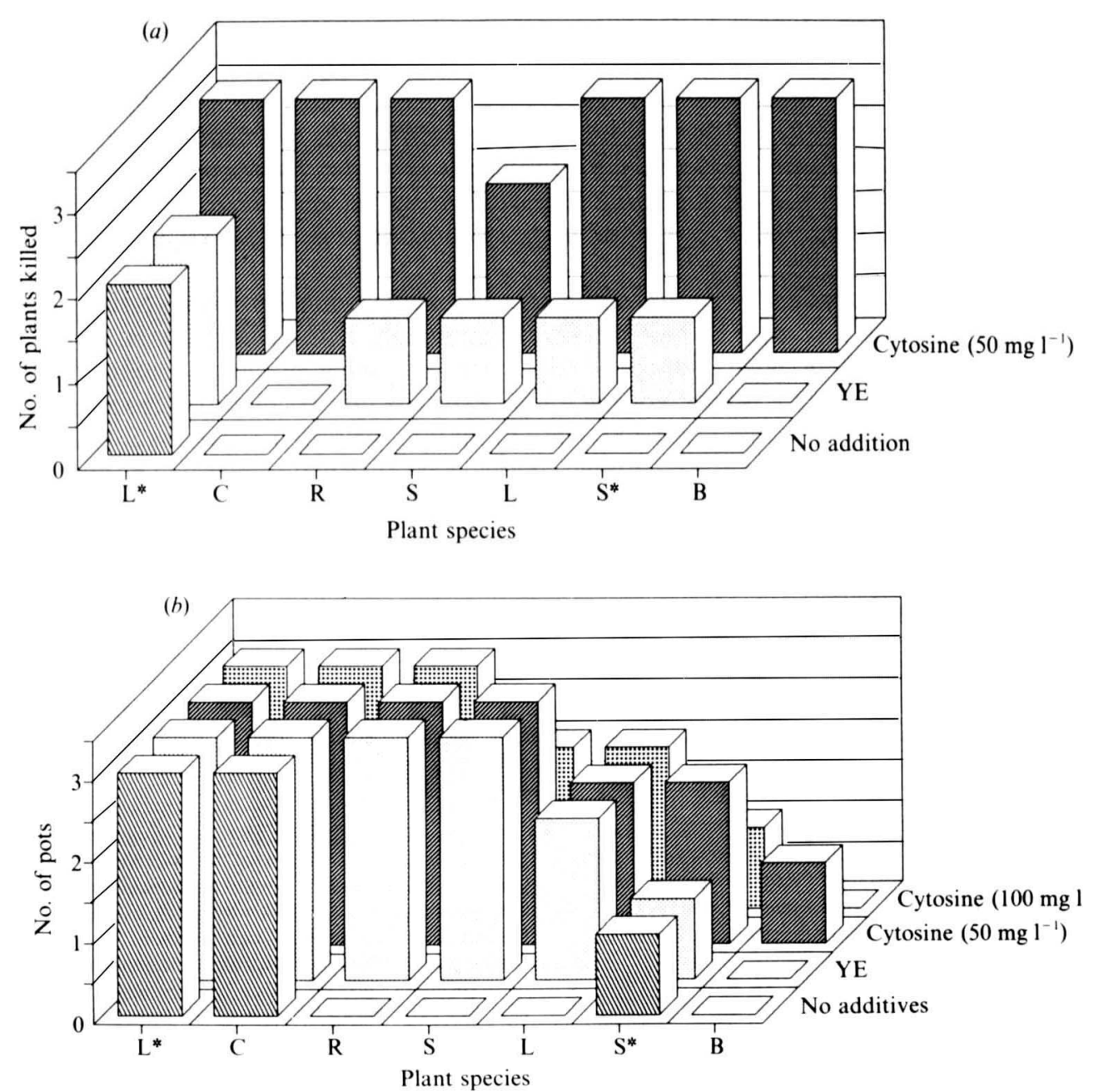

Fig. 4. Virulence of $S$. sclerotiorum A1-pyr on seven susceptible hosts from (a) PDA cultures with cotton plugs plus amendments applied to inoculation site, and $(b)$ infested millet with amendments added prior to inoculation. Amendments consisted of distilled water (control), $2 \mathrm{~g}$ yeast extract (YE) $\mathrm{I}^{-1}, 50 \mathrm{mg}$ cytosine $1^{-1}$ or $100 \mathrm{mg}$ cytosine $1^{-1}$. Cotton plugs were assessed on three replicate plants. Millet inoculations were assessed on replicate $15 \mathrm{~cm}$ diameter pots, each pot containing 10 lettuce, 10 clover, 10 rape, 3 safflower, 3 lentil, 2 sunflower, and 2 bean seedlings per pot (results are reported as number of pots in which the respective seedlings died). Plant species: B, bean (Phaseolus vulgaris L., cv. Bush Blue Lake 92); C, clover (Trifolium hybridum L., cv. Blossom); L, lentil (Lens culinaris Medik); L*, lettuce (Lactuca sativa L., cv. Grand Rapids); R, rape (Brassica napus L.); S, safflower (Carthamus tinctorus L., cv. S541); S*, sunflower (Helianthus annuus L., cv. D0-704).

\section{DISCUSSION}

In this study, an auxotrophic mutant of $S$. sclerotiorum, Al-pyr, was obtained by UV mutagenesis. The exact biochemical lesion in pyrimidine biosynthesis has not been determined. During initial isolation, Al-pyr was observed to grow more slowly than the wild-type on PDA. As opposed to some auxotrophs that can grow slowly on minimal agar, the cytosine requirement of A1-pyr is so complete that the mutant does not grow, from protoplasts, ascospores or mycelia, on minimal agar. 
A1-pyr is apparently the first auxotroph described for $S$. sclerotiorum, but auxotrophs have been reported with numerous other fungi (Boone et al., 1957, 1971; Garber et al., 1983, Kline et al., 1957; Leach et al., 1982; Lester et al., 1959; Perkins et al., 1982; Rowlands, 1983; Tinline, 1963; van Andel, 1966). Its cytosine requirement rendered A1-pyr avirulent on four of seven susceptible species of plants unless external cytosine was present in the inoculum or applied at the inoculation site. The association of avirulence with auxotrophy has been observed with other plant pathogens (Garber, 1954a, b; Garber et al., 1956; Kline et al., 1957; Tinline, 1963; van Andel, 1966).

Biological weed control, in both classical and mycoherbicide approaches, relies on organisms isolated from nature that must be host-specific (Charudattan, 1985; Templeton et al., 1979). Genetic manipulation of plant pathogens allows for improving biocontrol control agents (Charudattan, 1985). Such a genetic approach may also allow the use of pathogens such as $S$. sclerotiorum, by either increasing host-specificity or limiting pathogen dissemination. A1-pyr represents a possible candidate as a safer weed control agent. The pathogen would be limited to the area of application due to its auxotrophic requirement. As over $90 \%$ of apothecial stipes were aberrant, ascospore inoculum load would be negligible, particularly in the light of the relatively high abundance of the wild-type fungus in nature. Although A1-pyr must be field tested before conclusions can be reached on its feasibility for use as a biocontrol agent, this mutant may provide a method to control a number of important weeds.

This research was supported in part by a grant from the Montana Department of Agriculture in cooperation with the Missoula County Conservation District and Weed Board. We gratefully acknowledge Greg Kennett for support of this work. Contribution of the Montana Agricultural Experiment Station. Journal Series Paper J-2265.

\section{REFERENCES}

ADAMS, P. B. (1974). Host range of Whetzelinia sclerotiorum (Lib.) Korf and Dumont (Sclerotinia sclerotiorum (Lib.) deBary). Plant Protection Institute, Beltsville Agricultural Research Center - West, United States Department of Agriculture, Beltsville, Maryland.

Adams, P. B., Lumsden, R. D. \& TATe, C. J. (1974). Galinosoga paraflora: a new host for Whetzelinia sclerotiorum. Plant Disease Reporter 58, 700-701.

Adams, P. B., Marose, B. H. \& DutKy, E. M. (1983). Cocklebur: a new host for several Sclerotinia species. Plant Disease 67, 484-485.

vaN ANDEL, O. M. (1966). Amino acids and plant diseases. Annual Review of Phytopathology 4, 349368 .

BaCon, G. A., Burrows, T. W. \& Yates, M. (1951). The effects of biochemical mutation on the virulence of Bacterium typhosum: the loss of virulence of certain mutants. British Journal of Experimental Pathology 32, 85-96.

Blanchette, B. \& LeTourneau, D. (1977). The effects of gamma radiation on sclerotia of Whetzelinia sclerotiorum. Environmental and Experimental Botany 17, 49-54.

BoDINE, E. W. (1935). Sclerotinia wilt of Canada thistle. Phytopathology 25, 963-964.

BoONE, D. M. (1971). Genetics of Venturia inaequalis. Annual Review of Phytopathology 9, 297-318.

Boone, D. M., Kline, D. M. \& KeitT, G. W. (1957). Venturia inaequalis (Cke) Wint. XII. Pathogenicity of induced biochemical mutants. American Journal of Botany 44, 791-796.

Broston, B. S. \& SANDS, D. C. (1986). Field trials of Sclerotinia sclerotiorum to control Canada thistle (Cirsium arvense). Weed Science 34, 377-380.
Charudattan, R. (1985). The use of natural and genetically altered strains of pathogens for weed control. In Biological Control in Agricultural IPM Systems, pp. 347-372. Edited by M. A. Hoy \& D. C. Herzog. New York: Academic Press.

GARBER, E. D. (1954a). The role of nutrition in the host-parasite relationship. Proceedings of the National Academy of Sciences of the United States of America 40, 1112-1118.

GARBER, E. D. (1954b). A nutrition-inhibition hypothesis of pathogenicity. American Naturalist 90 , 183-194.

Garber, E. D., Shaeffer, S. G. \& Goldman, M. (1956). The virulence of biochemical mutants of Erwinia aroideae for varieties of radish and turnip. Journal of General Microbiology 14, 261-267.

Garber, R. C., Fry, W. E. \& Yoder, O. C. (1983). Conditional field epidemics on plants: a resource for research on population biology. Ecology 64, 16531655.

Garrabrandt, L. E., Johnson, S. A. \& Peterson, J. L. (1983). Tan sclerotia of Sclerotinia sclerotiorum from lettuce. Mycologia 75, 451-456.

Henson, L. \& Valleau, W. D. (1940). The production of apothecia of Sclerotinia sclerotiorum and $S$. trifoliorum in culture. Phytopathology 30, 869-873.

Holliday, R. (1956). A new method for the identification of biochemical mutants of micro-organisms. Nature, London 178, 987.

Huang, H. C. (1981). Tan sclerotia of Sclerotinia sclerotiorum. Canadian Journal of Plant Pathology 3, 136-138.

Huang, H. C. (1983). Pathogenicity and survival of the tan-sclerotial strain of Sclerotinia sclerotiorum. Canadian Journal of Plant Pathology 5, 245-247. 
Kline, D. M., Boone, D. M. \& KeITt, G. W. (1957). Venturia inaequalis (Cke.) Wint. XII. Nutritional control of pathogenicity of certain biochemical mutants. American Journal of Botany 44, 797-803.

KLISIEWICZK, J. M. (1986). Susceptibility of yellow star-thistle to selected plant pathogens. Plant Disease 70, 295-297.

Leach, J., LaNG, B. R. \& Yoder, O. C. (1982). Methods for selection of mutants and in vitro culture of Cochliobolus heterostrophus. Journal of General Microbiology 128, 1719-1729.

LESTER, H. E. \& Gross, S. R. (1959). Efficient method for selection of auxotrophic mutants of Neurospora. Science 129, 572.

LETOURNEAU, D. (1984). Inhibition of sclerotium formation by Sclerotinia sclerotionum with fluorophenylalanine. Transactions of the British Mycological Society 82, 156-159.

MoRGAN, O. D. (1971). A study of four weed hosts of Sclerotinia species in alfalfa fields. Plant Disease Reporter 55, 1087-1089.

Perkins, D. D., Radford, A., Newmeyer, D. \& Bjorkman, M. (1982). Chromosomal loci of Neurospora crassa. Microbiological Reviews 46, 426-570.

Price, K. \& Colhoun, J. (1975). Pathogenicity of isolates of Sclerotinia sclerotiorum (Lib.) deBary to several hosts. Phytopathologische Zeitschrift 83, 232 238.

PURDY, L. H. (1979). Sclerotinia sclerotiorum: history, diseases and symptomatology, host range, geographic distribution, and impact. Phytopathology 69, 875-880.
Rowlands, R. T. (1983). Industrial fungal genetics and strain selection. In The Filamentous Fungi, vol. 4, Fungal Technology, pp. 346-372. Edited by J. E. Smith, D. R. Berry \& B. Kristiansen. London: Edward Arnold.

SAITo, I. (1977). Studies on the maturation and germination of sclerotia of Sclerotinia sclerotiorum (Lib.) deBary, a causal fungus of bean stem rot. Report of the Hokkaido Prefectural Agricultural Experiment Station no. 26.

Stanway, C. A. \& Buck, K. W. (1984). Infection of protoplasts of the wheat take-all fungus Gaeumannomyces graminis var. tritici with double-stranded RNA viruses. Journal of General Virology 65, 2061-2066.

Templeton, G. E., TeBeest, D. O. \& SMith, R. J., JR (1979). Biological weed control with mycoherbicides. Annual Review of Phytopathology 17, 301-310.

TINLINE, R. D. (1963). Cochliobolus sativus. VII. Nutritional control of the pathogenicity of some auxotrophs to wheat seedlings. Canadian Journal of Botany 41, 489-497.

Watson, A. K., Copeman, R. J. \& Renney, A. J. (1974). A first record of Sclerotinia sclerotiorum and Microsphaeropsis centaureae on Centaurea diffusa. Canadian Journal of Botany 52, 2639-2640.

WoNG, A. L. \& WilleTTS, H. J. (1973). Electrophoretic studies of soluble proteins and enzymes of Sclerotinia species. Transactions of the British Mycological Society 61, 167-178.

WYLLIE, T. D. \& WALTER, J. M. (1957). Variation in Sclerotinia sclerotiorum induced by irradiation. Phytopathology 47, 538 (abstract). 